TRANSACTIONS OF THE

AMERICAN MATHEMATICAL SOCIETY

Volume 361, Number 11, November 2009, Pages 5967-5981

S 0002-9947(09)04909-5

Article electronically published on June 23, 2009

\title{
KÖNIG CHAINS FOR SUBMULTIPLICATIVE FUNCTIONS AND INFINITE PRODUCTS OF OPERATORS
}

\author{
JACEK JACHYMSKI
}

\begin{abstract}
We generalize the so-called Weighted König Lemma, due to Máté, for a submultiplicative function on a subset of the union $\bigcup_{n \in \mathbb{N}} \Sigma^{n}$, where $\Sigma$ is a set and $\Sigma^{n}$ is the Cartesian product of $n$ copies of $\Sigma$. Instead of a combinatorial argument as done by Máté, our proof uses Tychonoff's compactness theorem to show the existence of a König chain for a submultiplicative function. As a consequence, we obtain an extension of the Daubechies-Lagarias theorem concerning a finite set $\Sigma$ of matrices with right convergent products: Here we replace matrices by Banach algebra elements, and we substitute compactness for finiteness of $\Sigma$. The last result yields new generalizations of the KeliskyRivlin theorem on iterates of the Bernstein operators on the Banach space $C[0,1]$.
\end{abstract}

\section{INTRODUCTION}

Let $\Sigma$ be a set of square matrices (real or complex) of the same size. Following Daubechies and Lagarias DL92, we say that $\Sigma$ is a right convergent product set (in short, an $R C P$ set) if for any sequence $\left(M_{1}, M_{2}, \ldots\right) \in \Sigma^{\infty}, \lim _{n \rightarrow \infty} M_{1} M_{2} \cdots M_{n}$ exists. RCP sets of matrices arise in a number of different contexts such as constructing self-similar objects, the study of difference equations, probabilistic automata, wavelet analysis, interpolation schemes and nonhomogeneous Markov chains. (For more details, see [DL92] and the references therein.) In [DL92] necessary conditions and also some sufficient conditions are given for a set $\Sigma$ to be an RCP set. In particular, one of the main results of this paper ([DL92, Theorem 4.1]) says that a finite set $\Sigma$ is an RCP set whose limit function is identically zero if and only if $\hat{\rho}(\Sigma)<1$, where $\hat{\rho}(\Sigma)$ is the joint spectral radius of $\Sigma$. (This notion was introduced by Rota and Strang RS60 in a more general setting with $\Sigma$ being a bounded subset of a Banach algebra.) Subsequently, the DaubechiesLagarias theorem was generalized by Máté [Má98, Má99]. Using the ideas of the proof of [DL92, Theorem 3.1], he obtained a weighted version of the König Lemma for infinite trees ([Má98, Proposition 2], Má99, Theorem 1]) from which he derived a theorem [Má99, Theorem 2] giving a necessary and sufficient condition for the convergence of a submultiplicative function $\Phi$ to 0 . This function acts on some subset of $\bigcup_{n \in \mathbb{N}}\{1, \ldots, N\}^{n}$, the set of all blocks of the form $\left(\sigma_{1}, \ldots, \sigma_{n}\right)(n \in \mathbb{N}$,

Received by the editors October 5, 2007.

2000 Mathematics Subject Classification. Primary 47A35, 46H05, 47B38; Secondary 15A60, 26B35, 54D30, 54D20.

Key words and phrases. Submultiplicative function, joint spectral radius, König chain, Tychonoff's compactness theorem, infinite products of operators, Hutchinson system, Bernstein operators.

(c)2009 American Mathematical Society Reverts to public domain 28 years from publication 
$\left.\sigma_{1}, \ldots, \sigma_{n} \in\{1, \ldots, N\}\right)$. Then [DL92, Theorem 4.1] is a particular case of Má99, Theorem 2] with $\Phi$ defined as

$$
\Phi\left(\sigma_{1}, \ldots, \sigma_{n}\right):=\left\|M_{\sigma_{1}} \cdots M_{\sigma_{n}}\right\| \text { for } n \in \mathbb{N} \text { and } \sigma_{1}, \ldots, \sigma_{n} \in\{1, \ldots, N\},
$$

which is clearly submultiplicative in the sense that for $k, l \in \mathbb{N}$ and $\sigma_{1}, \ldots, \sigma_{k+l} \in$ $\{1, \ldots, N\}$,

$$
\Phi\left(\sigma_{1}, \ldots, \sigma_{k+l}\right) \leq \Phi\left(\sigma_{1}, \ldots, \sigma_{k}\right) \Phi\left(\sigma_{k+1}, \ldots, \sigma_{k+l}\right) .
$$

A generalization of the Daubechies-Lagarias theorem given by Shen [Sh00] goes in the other direction. Namely, he showed that, in general, results on finite RCP sets remained true for compact RCP sets. Among them there is the following one which is an important tool for studying RCP sets: For any matrix norm $\|\cdot\|$, a compact set $\Sigma$ of matrices has a König chain, i.e., a sequence $\left(M_{1}, M_{2}, \ldots\right) \in \Sigma^{\infty}$ such that

$$
\left\|M_{1} M_{2} \cdots M_{n}\right\|^{1 / n} \geq \hat{\rho}(\Sigma) \text { for each } n \in \mathbb{N} .
$$

(See [Sh00, Theorem 3]; for a finite $\Sigma$, this result can be spotted in the proof of DL92, Theorem 3.1].)

In this paper first we generalize and extend results of Máté [Má98, Má99 by considering submultiplicative functions on some subsets of the set $\bigcup_{n \in \mathbb{N}} \Sigma^{n}$ : Here $\Sigma$ is a compact topological space and $\Sigma^{n}$ is the Cartesian product of $n$ copies of $\Sigma$. We emphasize that the argument used in the proofs of [Má98, Proposition 2] and Má99, Theorem 1] cannot be carried over into this case, because this time the tree defined in [Má98, Má99] may have vertices of infinite degree unless $\Sigma$ is finite. Therefore, instead of the König Lemma, we apply a stronger tool-Tychonoff's compactness theorem. (It is known that the latter theorem implies the former.) In this way we obtain the result on the existence of a König chain for a submultiplicative function which, in turn, brings on a list of necessary and sufficient conditions for $\Phi$ to converge to 0 (cf. Theorem 4.2). The last result has a number of consequences which are discussed in Sections 5 and 6 . In particular, Theorem 5.1 extends the Daubechies-Lagarias result [DL92, Theorem 4.1]: Here we replace matrices by Banach algebra elements, and we substitute compactness for finiteness of $\Sigma$. Theorem 5.2 generalizes Máté's result [Má98. Theorem 1] concerning LCP (left convergent product) sets of linear bounded operators on a Hilbert space. This requires an independent analysis, though for matrices - as observed by Daubechies and Lagarias DL92] - it suffices to study RCP sets since $\Sigma$ is an LCP set if and only if $\Sigma^{t}$, the set of the transposes of all matrices from $\Sigma$, is an RCP set.

Finally, we analyse Hutchinson systems, the notion which originates from the theory of iterated function systems. Theorem 6.2 gives a necessary and sufficient condition for a family of linear bounded operators to be a Hutchinson system on every coset of a quotient space. As a consequence, we obtain a generalization of the Kelisky-Rivlin KR67 theorem on iterates of the Bernstein operators on the Banach space $C[0,1]$ (cf. Theorem 6.3).

\section{The RAdius of A SUBMUltiplicative FUnCTION}

We start with an auxiliary result on real sequences which, however, is not new. Nevertheless it seems some authors are unaware of that fact. Here we use a similar argument as in the proof of the existence of $\lim _{n \rightarrow \infty}\left\|T^{n}\right\|^{1 / n}$ for any linear bounded operator $T: X \rightarrow X$, where $X$ is a normed linear space (see, e.g., YYo80, p. 212]). The letter $\mathbb{R}_{+}$denotes the set of all nonnegative reals. 
Lemma 2.1. Let $\left(a_{n}\right)_{n \in \mathbb{N}}$ be a sequence of elements of $\mathbb{R}_{+} \cup\{\infty\}$ such that

$$
a_{n+m} \leq a_{n} a_{m} \text { for all } n, m \in \mathbb{N} \text {. }
$$

If $a_{1}<\infty$, then $a_{n}<\infty$ for all $n \in \mathbb{N}$, the sequence $\left(a_{n}^{1 / n}\right)_{n \in \mathbb{N}}$ is convergent and

$$
\lim _{n \rightarrow \infty} a_{n}^{1 / n} \leq a_{m}^{1 / m} \text { for each } m \in \mathbb{N} .
$$

Proof. By (2.1), we infer

$$
a_{n m}=a_{m+\cdots+m} \leq a_{m}^{n} \text { for } n, m \in \mathbb{N} .
$$

In particular, $a_{n} \leq a_{1}^{n}$. Hence, if $a_{1}$ is finite, so is $a_{n}$. Set

$$
a:=\inf \left\{a_{n}^{1 / n}: n \in \mathbb{N}\right\} .
$$

To complete the proof it suffices to show

$$
\limsup _{n \rightarrow \infty} a_{n}^{1 / n} \leq a .
$$

Given $\varepsilon>0$, there is $m \in \mathbb{N}$ such that $a_{m}^{1 / m}<a+\varepsilon$. Then, for $n \in \mathbb{N}$, there are $p_{n} \in \mathbb{N} \cup\{0\}$ and $q_{n} \in\{0, \ldots, m-1\}$ such that $n=p_{n} m+q_{n}$. By (2.1) and (2.2), we get

$$
a_{n}=a_{p_{n} m+q_{n}} \leq a_{p_{n} m} a_{q_{n}} \leq a_{m}^{p_{n}} a_{1}^{q_{n}}
$$

which yields

$$
a_{n}^{1 / n} \leq\left(a_{m}^{1 / m}\right)^{m p_{n} / n} a_{1}^{q_{n} / n} \leq(a+\varepsilon)^{1-q_{n} / n} a_{1}^{q_{n} / n} .
$$

Hence we obtain $\lim \sup _{n \rightarrow \infty} a_{n}^{1 / n} \leq a+\varepsilon$ since $q_{n} / n \rightarrow 0$. This yields (2.3) since $\varepsilon$ was an arbitrary positive real.

Remark 2.1. Lemma 2.1 can also be derived from a result of Pólya and Szegö (see, e.g., [HP57, Lemma 4.7.1]; here given without a proof) applied to the sequence $\left(\log a_{n}\right)_{n \in \mathbb{N}}$. (The case where $a_{k}=0$ for some $k \in \mathbb{N}$ is trivial.) In fact, both results are equivalent.

Throughout the paper $\Sigma$ is a nonempty set. For $n \in \mathbb{N}, \Sigma^{n}$ (resp., $\Sigma^{\infty}$ ) denotes the Cartesian product of $n$ (resp., countably many) copies of $\Sigma ; P_{(1, \ldots, n)}$ is the projection of $\Sigma^{\infty}$ onto $\Sigma^{n}$ defined by

$$
P_{(1, \ldots, n)}\left(\sigma_{1}, \sigma_{2}, \ldots\right):=\left(\sigma_{1}, \ldots, \sigma_{n}\right) \text { for }\left(\sigma_{m}\right)_{m \in \mathbb{N}} \in \Sigma^{\infty} .
$$

Let $s: \Sigma^{\infty} \rightarrow \Sigma^{\infty}$ be the shift operator, i.e.,

$$
s\left(\sigma_{1}, \sigma_{2}, \ldots\right):=\left(\sigma_{2}, \sigma_{3}, \ldots\right) \text { for }\left(\sigma_{m}\right)_{m \in \mathbb{N}} \in \Sigma^{\infty} .
$$

Let $\mathcal{K}$ be a shift-invariant subset of $\Sigma^{\infty}$, i.e., $s(\mathcal{K}) \subseteq \mathcal{K}$. Following Máté Má99, set

$$
B_{n}(\mathcal{K}):=P_{(1, \ldots, n)}(\mathcal{K}) \text { for } n \in \mathbb{N} \text {, and } B(\mathcal{K}):=\bigcup_{n \in \mathbb{N}} B_{n}(\mathcal{K}) .
$$

Thus $B_{n}(\mathcal{K})$ is the set of all $n$-tuples $\left(\sigma_{1}, \ldots, \sigma_{n}\right)$ obtained by truncation of $\sigma \in \mathcal{K}$. Since $\mathcal{K}$ is shift-invariant, it is easily seen that for any $n \geq 2$, if $\left(\sigma_{1}, \ldots, \sigma_{n}\right) \in B(\mathcal{K})$, then $\left(\sigma_{1}, \ldots, \sigma_{j}\right)$ and $\left(\sigma_{j+1}, \ldots, \sigma_{n}\right)$ are in $B(\mathcal{K})$ for any $j=1, \ldots, n-1$. This guarantees the correctness of the following definition taken from [Má99]. 
Definition 2.1. A function $\Phi: B(\mathcal{K}) \rightarrow \mathbb{R}_{+}$is said to be submultiplicative if for any $n \geq 2$ and $\left(\sigma_{1}, \ldots, \sigma_{n}\right) \in B_{n}(\mathcal{K})$,

$$
\Phi\left(\sigma_{1}, \ldots, \sigma_{n}\right) \leq \Phi\left(\sigma_{1}, \ldots, \sigma_{j}\right) \Phi\left(\sigma_{j+1}, \ldots, \sigma_{n}\right) \text { for } j=1, \ldots, n-1 .
$$

Remark 2.2. It is easy to show that Definition 2.1 has the following equivalent (and more natural) reformulation: $\Phi$ is submultiplicative if for any $v, w \in B(\mathcal{K})$,

$$
v w \in B(\mathcal{K}) \text { implies } \Phi(v w) \leq \Phi(v) \Phi(w),
$$

where $v w$ is the concatenation of $v$ and $w$. In this case we need not assume $\mathcal{K}$ is shiftinvariant; however, this assumption is necessary for the correctness of Definition 2.2 given below.

Lemma 2.2. Let $\Phi: B(\mathcal{K}) \rightarrow \mathbb{R}_{+}$be submultiplicative and such that $\left.\Phi\right|_{B_{1}(\mathcal{K})}$ is bounded. For $n \in \mathbb{N}$, set

$$
\rho_{n}(\Phi):=\sup \left\{\Phi(w): w \in B_{n}(\mathcal{K})\right\} .
$$

Then each $\rho_{n}(\Phi)$ is finite, the sequence $\left(\rho_{n}^{1 / n}(\Phi)\right)_{n \in \mathbb{N}}$ is convergent and

$$
\lim _{n \rightarrow \infty} \rho_{n}^{1 / n}(\Phi) \leq \rho_{m}^{1 / m}(\Phi) \text { for each } m \in \mathbb{N} .
$$

Proof. Clearly, $\rho_{1}(\Phi)$ is finite. Let $n, m \in \mathbb{N}$ and $w \in B_{n+m}(\mathcal{K})$. By hypothesis,

$$
\Phi(w)=\Phi\left(\sigma_{1}, \ldots, \sigma_{n+m}\right) \leq \Phi\left(\sigma_{1}, \ldots, \sigma_{n}\right) \Phi\left(\sigma_{n+1}, \ldots, \sigma_{n+m}\right) \leq \rho_{n}(\Phi) \rho_{m}(\Phi) .
$$

Hence we infer $\rho_{n+m}(\Phi) \leq \rho_{n}(\Phi) \rho_{m}(\Phi)$, so it suffices to apply Lemma 2.1.

Definition 2.2. For a function $\Phi$ as in Lemma 2.2, the number

$$
\rho(\Phi):=\lim _{n \rightarrow \infty} \rho_{n}^{1 / n}(\Phi)
$$

is called the radius of $\Phi$. Then $\rho(\Phi) \leq \rho_{n}^{1 / n}(\Phi)$ for each $n \in \mathbb{N}$.

Remark 2.3. Máté [Má98, Má99] considered submultiplicative functions only for the case where $\Sigma$ is finite. Moreover, in Má98 he defined the radius of $\Phi$ as $\lim \sup _{n \rightarrow \infty} \rho_{n}^{1 / n}(\Phi)$. limsup also occurs in the Daubechies-Lagarias DL92] definition of the joint spectral radius $\hat{\rho}(\Sigma)$ of a set of matrices; in DL01 they observed that limsup could be replaced by lim (see [DL01, Lemma 3.1 and Remark]). Máté Má99 also substituted lim for limsup in the definition of $\rho(\Phi)$ (without giving any grounds), and he obtained the inequality $\rho(\Phi) \leq \rho_{n}^{1 / n}(\Phi)$, but it was done in a nontrivial way - with the help of the König Lemma (see [Má99, Corollary]).

\section{KÖNIG CHAINS FOR SEQUENCES OF SETS}

We extend Shen's [Sh00] notion of a König chain for a set of matrices (cf. [1.1)) in the following way. Let $\Sigma$ be an arbitrary set and for $n \in \mathbb{N}$, let $X_{n}$ be a subset of $\Sigma^{n}$.

Definition 3.1. A sequence $\left(\sigma_{1}, \sigma_{2}, \ldots\right) \in \Sigma^{\infty}$ is a König chain for $\left(X_{n}\right)_{n \in \mathbb{N}}$ if

$$
\left(\sigma_{1}, \ldots, \sigma_{n}\right) \in X_{n} \text { for } n \in \mathbb{N} .
$$

We start with a topological result on the existence of a König chain. Here the Cartesian products $\Sigma^{n}$ and $\Sigma^{\infty}$ are endowed with the Tychonoff topology. 
Proposition 3.1. Let $\Sigma$ be a compact topological space and let $\mathcal{K}$ be a closed subset of $\Sigma^{\infty}$. For each $n \in \mathbb{N}$, let $\left(X_{n m}\right)_{m \in \mathbb{N}}$ be a descending sequence of closed subsets of $\Sigma^{n}$, and $X_{n}:=\bigcap_{m \in \mathbb{N}} X_{n m}$. The following statements are equivalent:

(i) there exists a König chain for $\left(X_{n}\right)_{n \in \mathbb{N}}$ which belongs to $\mathcal{K}$;

(ii) given $n \in \mathbb{N}$, there is $\sigma \in \mathcal{K}$ such that $\left(\sigma_{1}, \ldots, \sigma_{k}\right) \in X_{k}$ for $k=1, \ldots, n$;

(iii) given $m, n \in \mathbb{N}$, there is $\sigma \in \mathcal{K}$ such that $\left(\sigma_{1}, \ldots, \sigma_{k}\right) \in X_{k m}$ for $k=$ $1, \ldots, n$.

Proof. (i) $\Rightarrow$ (iii) is obvious. (iii) $\Rightarrow($ ii): Fix $n \in \mathbb{N}$, and for $m \in \mathbb{N}$, set

$$
F_{m}:=\mathcal{K} \cap \bigcap_{k=1}^{n} P_{(1, \ldots, k)}^{-1}\left(X_{k m}\right) .
$$

By (iii), $F_{m} \neq \emptyset$. Since for $k=1, \ldots, n,\left(X_{k m}\right)_{m \in \mathbb{N}}$ is descending, so is $\left(F_{m}\right)_{m \in \mathbb{N}}$. Moreover, all $F_{m}$ are closed because of the continuity of $P_{(1, \ldots, k)}$ and the closeness of $\mathcal{K}$ and $X_{k m}$ for $k=1, \ldots, n$. By Tychonoff's theorem, $\Sigma^{\infty}$ is compact; in particular, it is countably compact. Hence $\bigcap_{m \in \mathbb{N}} F_{m} \neq \emptyset$. It is easily seen that if $\sigma \in \bigcap_{m \in \mathbb{N}} F_{m}$, then $\sigma \in \mathcal{K}$ and $\left(\sigma_{1}, \ldots, \sigma_{k}\right) \in X_{k}$ for $k=1, \ldots, n$, so (ii) holds.

(ii) $\Rightarrow(\mathrm{i})$ : For $n \in \mathbb{N}$, set

$$
F_{n}:=\mathcal{K} \cap \bigcap_{k=1}^{n} P_{(1, \ldots, k)}^{-1}\left(X_{k}\right) .
$$

By (ii), $F_{n} \neq \emptyset$. Clearly, $\left(F_{n}\right)_{n \in \mathbb{N}}$ is a descending sequence of closed sets, so by countable compactness of $\Sigma^{\infty}, \bigcap_{n \in \mathbb{N}} F_{n} \neq \emptyset$. Then any $\sigma \in \bigcap_{n \in \mathbb{N}} F_{n}$ is a König chain for $\left(X_{n}\right)_{n \in \mathbb{N}}$.

Remark 3.1. In fact, the proof of Proposition 3.1 shows that the assumption on $\Sigma$ can be weakened: It suffices that $\Sigma$ is a topological space such that $\Sigma^{\infty}$ is countably compact. (This forces the countable compactness of $\Sigma$.) In particular, this is the case if $\Sigma$ is compact or sequentially compact (see, e.g., En77, Theorems 3.10.35 and 3.10.30]); in the latter case the Tychonoff theorem is unnecessary. For a more detailed discussion - when is a product of countably compact spaces countably compact? - see [Va84.

The referee suggested finding some nontrivial examples of sequences $\left(X_{n}\right)_{n \in \mathbb{N}}$ without König chains. Actually, such sequences exist for any topological space $\Sigma$ which is not countably compact, in accordance with the following.

Proposition 3.2. Let $\Sigma$ be a topological space which is not countably compact. Then there exists a sequence $\left(X_{n}\right)_{n \in \mathbb{N}}$ without König chains satisfying (ii) of Proposition 3.1 with $\mathcal{K}:=\Sigma^{\infty}$, and such that each $X_{n}$ is a closed subset of $\Sigma^{n}$.

Proof. By hypothesis, there exists a descending sequence $\left(A_{n}\right)_{n \in \mathbb{N}}$ of nonempty closed subsets of $\Sigma$ such that $\bigcap_{n \in \mathbb{N}} A_{n}=\emptyset$. Set

$$
X_{1}:=A_{1} \text { and } X_{n}:=A_{n} \times A_{n-1} \times \cdots \times A_{1} \text { for } n \geq 2 .
$$

Clearly, each $X_{n}$ is a nonempty closed subset of $\Sigma^{n}$. Let $n \in \mathbb{N}$ and $\left(\sigma_{1}, \ldots, \sigma_{n}\right) \in$ $X_{n}$. Since $\left(A_{n}\right)_{n \in \mathbb{N}}$ is descending, we get $\left(\sigma_{1}, \ldots, \sigma_{k}\right) \in X_{k}$ for $k=1, \ldots, n$. So setting $\sigma:=\left(\sigma_{1}, \ldots, \sigma_{n}, \sigma_{n}, \ldots\right)$, we infer (ii) of Proposition 3.1 holds. Suppose, on the contrary, there is a König chain $\left(\sigma_{n}\right)_{n \in \mathbb{N}}$ for $\left(X_{n}\right)_{n \in \mathbb{N}}$. Then for any $n \in \mathbb{N}$, $\left(\sigma_{1}, \ldots, \sigma_{n}\right) \in X_{n}$ and hence $\sigma_{1} \in \bigcap_{n \in \mathbb{N}} A_{n}=\emptyset$, a contradiction. 
As an immediate consequence of Propositions 3.1] and 3.2, we get the following.

Corollary 3.1. Let $\Sigma$ be a Lindelöf topological space or a metric space. The following statements are equivalent:

(i) $\Sigma$ is compact;

(ii) any sequence $\left(X_{n}\right)_{n \in \mathbb{N}}$ satisfying (ii) of Proposition 3.1 with $\mathcal{K}:=\Sigma^{\infty}$, and such that each $X_{n}$ is a closed subset of $\Sigma^{n}$, has a König chain.

Our next result is of an algebraic nature and it gives a sufficient condition for (iii) of Proposition 3.1 to hold. We say that the concatenation is conditionally internal in a subset $B$ of $B(\mathcal{K})$ if for any $v, w \in B, v w \in B(\mathcal{K})$ implies $v w \in B$.

Proposition 3.3. Let $\Sigma$ be a set, and $\mathcal{K}$ be a shift-invariant subset of $\Sigma^{\infty}$. For $n, m \in \mathbb{N}$, let $X_{n m}$ be a subset of $B_{n}(\mathcal{K})$ and $Y_{n m}:=B_{n}(\mathcal{K}) \backslash X_{n m}$. Assume that the following two conditions hold:

(A) for any $n \in \mathbb{N}, \bigcup_{m \in \mathbb{N}} Y_{n m} \neq B_{n}(\mathcal{K})$;

(B) for any $m \in \mathbb{N}$, the concatenation is conditionally internal in the set $\bigcup_{n \in \mathbb{N}} Y_{n m}$ and given $r \in \mathbb{N}$, there is $p \in \mathbb{N}$ such that for any $w \in B_{r}(\mathcal{K})$ and $v \in Y_{n m}$ with $n \geq p$, if $v w \in B(\mathcal{K})$, then $v w \in Y_{n+r, j}$ for some $j \in \mathbb{N}$.

Then, given $m, n \in \mathbb{N}$, there is $\sigma \in \mathcal{K}$ such that $\left(\sigma_{1}, \ldots, \sigma_{k}\right) \in X_{k m}$ for $k=1, \ldots, n$.

Remark 3.2. Roughly speaking the second part of (B) says that the concatenation of any block and a sufficiently long block from any set of the distinguished family is to belong to some set of that family.

Proof of Proposition 3.3. Fix $m \in \mathbb{N}$ and set

$$
Z_{n}:=\left\{\sigma \in \mathcal{K}:\left(\sigma_{1}, \ldots, \sigma_{k}\right) \in X_{k m} \text { for } k=1, \ldots, n\right\} .
$$

We are to show $Z_{n} \neq \emptyset$ for all $n \in \mathbb{N}$. Suppose, on the contrary,

$$
q:=\min \left\{n \in \mathbb{N}: Z_{n}=\emptyset\right\} .
$$

By $(\mathrm{A}), B_{1}(\mathcal{K}) \backslash \bigcup_{j \in \mathbb{N}} Y_{1 j} \neq \emptyset$, i.e., $\bigcap_{j \in \mathbb{N}} X_{1 j} \neq \emptyset$. In particular, $X_{1 m} \neq \emptyset$, which yields $Z_{1} \neq \emptyset$, i.e., $q \geq 2$.

Let $\sigma \in \mathcal{K}$ and $n \geq q$.

Claim. $\left(\sigma_{1}, \ldots, \sigma_{n}\right)$ is the concatenation of a block $v \in \bigcup_{k \in \mathbb{N}} Y_{k m}$ and a block $w \in \bigcup_{k=1}^{q-1} B_{k}(\mathcal{K}) \cup\{\emptyset\}$ (here the sign $\emptyset$ denotes the empty block).

Clearly, for any $j \in \mathbb{N}, B_{j}(\mathcal{K})=X_{j m} \cup Y_{j m}$, so $\left(\sigma_{1}, \ldots, \sigma_{j}\right) \in X_{j m} \cup Y_{j m}$. If $\sigma_{1} \in Y_{1 m}$, we set $v^{(1)}:=\sigma_{1}$. If $\sigma_{1} \notin Y_{1 m}$ (i.e., $\left.\sigma_{1} \in X_{1 m}\right)$ and $\left(\sigma_{1}, \sigma_{2}\right) \in Y_{2 m}$, we set $v^{(1)}:=\left(\sigma_{1}, \sigma_{2}\right)$; otherwise, we look at $\left(\sigma_{1}, \sigma_{2}, \sigma_{3}\right)$. Continuing in this fashion, we obtain after at most $q$ steps (since $Z_{q}=\emptyset$ ) that $v^{(1)} \in \bigcup_{k=1}^{q} Y_{k m}$. Then $\left(\sigma_{1}, \ldots, \sigma_{n}\right)=v^{(1)} w^{(1)}$. Let $|\cdot|$ denote the length of a block. If $\left|w^{(1)}\right| \geq q$, then we repeat the above argument (note that $w^{(1)} \in B_{\left|w^{(1)}\right|}(\mathcal{K})$ since $\mathcal{K}$ is shift-invariant) to get $w^{(1)}=v^{(2)} w^{(2)}$, where $v^{(2)} \in \bigcup_{k=1}^{q} Y_{k m}$. In this way we may infer $\left(\sigma_{1}, \ldots, \sigma_{n}\right)$ is the concatenation of some blocks from $\bigcup_{k=1}^{q} Y_{k m}$ and a block from $\bigcup_{k=1}^{q-1} B_{k}(\mathcal{K}) \cup\{\emptyset\}$ which, in view of the first part of (B), proves the claim.

By (B), for $r=1, \ldots, q-1$, there exist appropriate integers $p_{1}, \ldots, p_{q-1}$. Set

$$
p:=\max \left\{p_{1}, \ldots, p_{q-1}\right\} \text { and } n:=p+q-1 .
$$

By (A), there is $\sigma \in \mathcal{K}$ such that $\left(\sigma_{1}, \ldots, \sigma_{n}\right) \notin \bigcup_{j \in \mathbb{N}} Y_{n j}$. Since $n \geq q$, in view of the claim there are $v \in \bigcup_{k \in \mathbb{N}} Y_{k m}$ and $w \in B_{r}(\mathcal{K}) \cup\{\emptyset\}$ for some $r \in\{1, \ldots, q-1\}$ 
such that $\left(\sigma_{1}, \ldots, \sigma_{n}\right)=v w$. In fact, $w \neq \emptyset$; otherwise, $\left(\sigma_{1}, \ldots, \sigma_{n}\right) \in Y_{n m}$, a contradiction. Moreover,

$$
|v|=n-|w| \geq n-q+1=p \geq p_{r} .
$$

Thus we have $w \in B_{r}(\mathcal{K}), v \in Y_{|v| m},|v| \geq p_{r}$ and $v w \in B(\mathcal{K})$, so by the property of $p_{r}, v w \in Y_{|v|+r, m}$, i.e., $\left(\sigma_{1}, \ldots, \sigma_{n}\right) \in Y_{n m}$, which yields a contradiction.

Remark 3.3. The referee pointed out that Proposition 3.3 extends Má99, Proposition 1] since it is easily seen that if $\Sigma$ is finite, then the condition that each $Z_{n}$ defined by (3.1) is nonempty is equivalent to the fact that the set

$\mathcal{T}:=\left\{v \in B(\mathcal{K}): \exists_{n \in \mathbb{N}}\left(v=\left(v_{1}, \ldots, v_{n}\right)\right.\right.$ and $\left(v_{1}, \ldots, v_{k}\right) \in X_{k m}$ for $\left.\left.k=1, \ldots, n\right)\right\}$

considered in [Má99] is infinite. But even for a finite $\Sigma$, Proposition 3.3 is still more general than [Má99, Proposition 1] since the latter result was proved for some particular sets $X_{k m}$.

\section{KÖNIG CHAINS FOR SUBMULTIPLICATIVE FUNCTIONS}

Let $\Phi: B(\mathcal{K}) \rightarrow \mathbb{R}_{+}$be submultiplicative (cf. Definition 2.1) and such that $\left.\Phi\right|_{B_{1}(\mathcal{K})}$ is bounded. Let $\rho(\Phi)$ denote the radius of $\Phi$ (cf. Definition 2.2).

Definition 4.1. A sequence $\left(\sigma_{1}, \sigma_{2}, \ldots\right) \in \mathcal{K}$ is a König chain for $\Phi$ if

$$
\Phi\left(\sigma_{1}, \ldots, \sigma_{n}\right) \geq \rho^{n}(\Phi) \text { for } n \in \mathbb{N} .
$$

The following result is a generalization of [Má98, Proposition 2] and Má99, Theorem 1]: Instead of finiteness of a set $\Sigma$, we need $\Sigma$ to be compact.

Theorem 4.1. Let $\Sigma$ be a compact topological space and $\mathcal{K}$ be a nonempty closed and shift-invariant subset of $\Sigma^{\infty}$. Let $\Phi: B(\mathcal{K}) \rightarrow \mathbb{R}_{+}$be submultiplicative and such that for any $n \in \mathbb{N},\left.\Phi\right|_{B_{n}(\mathcal{K})}$ is upper semicontinuous. Then there exists a König chain for $\Phi$.

Proof. Since by Tychonoff's theorem, $\Sigma^{\infty}$ is compact, so is $\mathcal{K}$. Hence, given $n \in \mathbb{N}$, $B_{n}(\mathcal{K})$ is compact. Since $\left.\Phi\right|_{B_{n}(\mathcal{K})}$ is upper semicontinuous, it is bounded, so by Lemma 2.2. the radius $\rho:=\rho(\Phi)$ is well-defined. Moreover, $\left.\Phi\right|_{B_{n}(\mathcal{K})}$ attains its supremum, so there is $w_{n} \in B_{n}(\mathcal{K})$ such that $\Phi\left(w_{n}\right)=\rho_{n}(\Phi)$ (cf. (2.4)). Hence and by Lemma 2.2, we infer the set

$$
X_{n}:=\left\{w \in B_{n}(\mathcal{K}): \Phi(w) \geq \rho^{n}\right\}
$$

is nonempty. With the help of Propositions 3.1 and 3.3 we will show there is a König chain in $\mathcal{K}$ for $\left(X_{n}\right)_{n \in \mathbb{N}}$ (hence, also for $\Phi$ since the two notions coincide). In the sequel we assume $\rho>0$; otherwise, every $\sigma \in \mathcal{K}$ is a König chain for $\Phi$. For $m, n \in \mathbb{N}$, set

$$
X_{n m}:=\left\{w \in B_{n}(\mathcal{K}): \Phi(w) \geq \rho^{n}(1-1 /(m+1))^{n}\right\} .
$$

It is clear that for $n \in \mathbb{N},\left(X_{n m}\right)_{m \in \mathbb{N}}$ is descending and $X_{n}=\bigcap_{m \in \mathbb{N}} X_{n m}$. Moreover, all $X_{n m}$ are closed because of the upper semicontinuity of $\left.\Phi\right|_{B_{n}(\mathcal{K})}$. Thus it suffices to show that (iii) of Proposition 3.1 holds. To do that, we will apply Proposition 3.3. So let $Y_{n m}:=B_{n}(\mathcal{K}) \backslash X_{n m}$ for $m, n \in \mathbb{N}$. Then

$$
Y_{n m}=\left\{w \in B_{n}(\mathcal{K}): \Phi(w)<\rho^{n}(1-1 /(m+1))^{n}\right\} .
$$


Since $X_{n} \neq \emptyset$, i.e., $\bigcap_{m \in \mathbb{N}} X_{n m} \neq \emptyset$, (A) of Proposition 3.3 holds. To verify (B), fix $m \in \mathbb{N}$. Given $j, k \in \mathbb{N}$, if $v \in Y_{j m}, w \in Y_{k m}$ and $v w \in B(\mathcal{K})$, then

$$
\Phi(v w) \leq \Phi(v) \Phi(w)<\rho^{j+k}(1-1 /(m+1))^{j+k},
$$

which means that $v w \in Y_{j+k, m}$. Now let $r \in \mathbb{N}$. Since

$$
\lim _{n \rightarrow \infty} \frac{\rho^{r}}{(1-1 /(m+1))^{n}}=\infty,
$$

there is $p \in \mathbb{N}$ such that for $n \geq p, \rho^{r} /(1-1 /(m+1))^{n}>\rho_{r}(\Phi)$. Now, if $w \in B_{r}(\mathcal{K})$, $v \in Y_{n m}$ with $n \geq p$, and $v w \in B(\mathcal{K})$, then

$$
\Phi(v w) \leq \Phi(v) \Phi(w)<\rho^{n}(1-1 /(m+1))^{n} \rho_{r}(\Phi)<\rho^{r+n},
$$

which means that $v w \in B_{n+r}(\mathcal{K}) \backslash X_{n+r}=\bigcup_{j \in \mathbb{N}} Y_{n+r, j}$. Thus (B) holds, so Proposition 3.3 is applicable.

Remark 4.1. Clearly, if $\Sigma$ is finite, so is $\Sigma^{n}$. Hence $B_{n}(\mathcal{K})\left(\subseteq \Sigma^{n}\right)$ is finite, which implies the continuity of $\left.\Phi\right|_{B_{n}(\mathcal{K})}$. So Theorem 4.1] does extend [Má98, Proposition 2] and [Má99, Theorem 1]. The referee observed that in the case in which $\Sigma$ is a compact metrizable space, the proof of Theorem 4.1 could be shortened by using Máté's theorem [Má99, Theorem 1] and an argument involving finite $\varepsilon$-nets. Moreover, for a finite $\Sigma$, the above proof can be simplified: There is no need to introduce sets $X_{n m}$ and it suffices to apply the first part of Proposition 3.1 ((ii) $\Rightarrow(\mathrm{i})$ ).

As a consequence, we get the following extension of [Má99, Theorem 2].

Theorem 4.2. Under the assumptions of Theorem 4.1, the following statements are equivalent:

(i) $\rho(\Phi)<1$;

(ii) for any $\sigma \in \mathcal{K}$, the series $\sum_{n=1}^{\infty} \Phi\left(\sigma_{1}, \ldots, \sigma_{n}\right)$ converges to some $S(\sigma) \in \mathbb{R}_{+}$, and $S_{n}(\sigma) \rightarrow S(\sigma)$ uniformly with respect to $\sigma \in \mathcal{K}$, where $S_{n}(\sigma)$ is the nth partial sum of the above series;

(iii) $\lim _{n \rightarrow \infty} \Phi\left(\sigma_{1}, \ldots, \sigma_{n}\right)=0$ uniformly with respect to $\sigma \in \mathcal{K}$;

(iv) for any $\sigma \in \mathcal{K}, \lim _{n \rightarrow \infty} \Phi\left(\sigma_{1}, \ldots, \sigma_{n}\right)=0$;

(v) for any $\sigma \in \mathcal{K}, \liminf _{n \rightarrow \infty} \Phi\left(\sigma_{1}, \ldots, \sigma_{n}\right)<1$.

Proof. (i) $\Rightarrow$ (ii): Since $\rho_{n}^{1 / n}(\Phi) \rightarrow \rho(\Phi)<1$, there are $\alpha \in(0,1)$ and $k \in \mathbb{N}$ such that $\rho_{n}(\Phi) \leq \alpha^{n}$ for $n \geq k$. Set

$$
f_{n}(\sigma):=\Phi\left(\sigma_{1}, \ldots, \sigma_{n}\right) \text { for } \sigma \in \mathcal{K} \text { and } n \in \mathbb{N} .
$$

Then $f_{n}(\sigma) \leq \alpha^{n}$ for $n \geq k$ and $\sigma \in \mathcal{K}$, so the Weierstrass criterion yields the uniform convergence of $\sum_{n=1}^{\infty} f_{n}(\sigma)$.

The implications (ii) $\Rightarrow$ (iii) $\Rightarrow$ (iv) $\Rightarrow$ (v) are obvious.

$(\mathrm{v}) \Rightarrow(\mathrm{i})$ : Suppose, on the contrary, $\rho(\Phi) \geq 1$. By Theorem 4.1, there exists a König chain $\sigma$ for $\Phi$, i.e.,

$$
\Phi\left(\sigma_{1}, \ldots, \sigma_{n}\right) \geq \rho^{n}(\Phi) \geq 1 \text { for } n \in \mathbb{N},
$$

which yields $\liminf \operatorname{in}_{n \rightarrow \infty} \Phi\left(\sigma_{1}, \ldots, \sigma_{n}\right) \geq 1$, a contradiction. 


\section{INFINITE PRODUCTS OF BANACH ALGEBRA ELEMENTS}

Let $\Sigma$ be a bounded subset of a Banach algebra. Recall (cf. [RS60]) that the Rota-Strang joint spectral radius of $\Sigma$ is defined by

$$
\hat{\rho}(\Sigma):=\lim _{n \rightarrow \infty} \sup \left\{\left\|\sigma_{1} \cdots \sigma_{n}\right\|^{1 / n}: \sigma_{1}, \ldots, \sigma_{n} \in \Sigma\right\} .
$$

If we set

$$
\Phi\left(\sigma_{1}, \ldots, \sigma_{n}\right):=\left\|\sigma_{1} \cdots \sigma_{n}\right\| \text { for } n \in \mathbb{N} \text { and } \sigma_{1}, \ldots, \sigma_{n} \in \Sigma,
$$

then $\Phi: \bigcup_{n \in \mathbb{N}} \Sigma^{n} \rightarrow \mathbb{R}_{+}$is submultiplicative and $\left.\Phi\right|_{\Sigma^{n}}$ is continuous for any $n \in \mathbb{N}$. Moreover, the monotonicity and continuity of $t \mapsto t^{1 / n}\left(t \in \mathbb{R}_{+}\right)$yield $\hat{\rho}(\Sigma)=\rho(\Phi)$. Thus, applying Theorem 4.2, we easily get the following generalization of [DL92, Theorem 4.1] and [Sh00, Proposition 2]. The novelty here is that we substitute Banach algebra elements for matrices, and we extend a list of necessary and sufficient conditions for the convergence of all infinite products to 0 .

Theorem 5.1. Let $\Sigma$ be a compact subset of a Banach algebra. The following statements are equivalent:

(i) $\hat{\rho}(\Sigma)<1$;

(ii) for any $\sigma=\left(\sigma_{n}\right)_{n \in \mathbb{N}} \in \Sigma^{\infty}$, the series $\sum_{n=1}^{\infty} \sigma_{1} \cdots \sigma_{n}$ is absolutely convergent, and the set $C:=\left\{\sum_{n=1}^{\infty} \sigma_{1} \cdots \sigma_{n}: \sigma \in \Sigma^{\infty}\right\}$ is compact;

(iii) for any $\sigma \in \Sigma^{\infty}$, the series $\sum_{n=1}^{\infty} \sigma_{1} \cdots \sigma_{n}$ is convergent;

(iv) $\lim _{n \rightarrow \infty} \sigma_{1} \cdots \sigma_{n}=0$ uniformly with respect to $\sigma \in \Sigma^{\infty}$;

(v) for any $\sigma \in \Sigma^{\infty}, \lim _{n \rightarrow \infty} \sigma_{1} \cdots \sigma_{n}=0$;

(vi) for any $\sigma \in \Sigma^{\infty}, \lim _{n \rightarrow \infty} \sigma_{n} \cdots \sigma_{1}=0$;

(vii) for any $\sigma \in \Sigma^{\infty}, \liminf _{n \rightarrow \infty}\left\|\sigma_{1} \cdots \sigma_{n}\right\|<1$.

Proof. We apply Theorem 4.2 with $\mathcal{K}:=\Sigma^{\infty}$. Then $B_{n}(\mathcal{K})=\Sigma^{n}$ and $B(\mathcal{K})=\Sigma^{*}$, where $\Sigma^{*}:=\bigcup_{n \in \mathbb{N}} \Sigma^{n}$, the set of all finite sequences of elements of $\Sigma$. We consider $\Phi: \Sigma^{*} \rightarrow \mathbb{R}_{+}$defined by (5.1). By Theorem 4.2, (i), (iv), (v) and (vii) are equivalent. By completeness, (ii) implies (iii). (iii) $\Rightarrow(\mathrm{v})$ is clear. Thus it suffices to show (i) $\Rightarrow$ (ii) and (i) $\Leftrightarrow$ (vi).

(i) $\Rightarrow$ (ii): The absolute convergence of $\sum_{n=1}^{\infty} \sigma_{1} \cdots \sigma_{n}$ follows from Theorem 4.2 ((i) $\Rightarrow($ ii) $)$. We show $C$ is compact. For $\sigma \in \Sigma^{\infty}$ and $n \in \mathbb{N}$, set

$$
S_{n}(\sigma):=\sum_{k=1}^{n} \sigma_{1} \cdots \sigma_{k} \text { and } S(\sigma):=\sum_{k=1}^{\infty} \sigma_{1} \cdots \sigma_{k} .
$$

Then $\left\|S_{n}(\sigma)-S(\sigma)\right\| \leq \sum_{k=n+1}^{\infty} \Phi\left(\sigma_{1}, \ldots, \sigma_{k}\right) \rightarrow 0$ as $n \rightarrow \infty$, uniformly with respect to $\sigma \in \Sigma^{\infty}$ in view of Theorem $4.2((\mathrm{i}) \Rightarrow(\mathrm{ii}))$. Hence $\left(S_{n}\right)_{n \in \mathbb{N}}$ converges to $S$ uniformly on $\Sigma^{\infty}$. Since each $S_{n}$ is continuous on $\Sigma^{\infty}$, so is $S$. Since $\Sigma^{\infty}$ is compact and $C=S\left(\Sigma^{\infty}\right)$, we infer $C$ is compact.

To show (i) $\Leftrightarrow\left(\right.$ vi), define $\Psi: \Sigma^{*} \rightarrow \mathbb{R}_{+}$by

$$
\Psi\left(\sigma_{1}, \ldots, \sigma_{n}\right):=\left\|\sigma_{n} \cdots \sigma_{1}\right\| \text { for } n \in \mathbb{N} \text { and } \sigma_{1}, \ldots, \sigma_{n} \in \Sigma .
$$

Clearly, $\Psi$ satisfies the assumptions of Theorem 4.2. Hence $\rho(\Psi)<1$ if and only if $\lim _{n \rightarrow \infty} \Psi\left(\sigma_{1}, \ldots, \sigma_{n}\right)=0$ for $\sigma \in \Sigma^{\infty}$. Since $\rho(\Psi)=\rho(\Phi)=\hat{\rho}(\Sigma)$, we infer (i) and (vi) are equivalent.

Remark 5.1. The version of Theorem 5.1 - for a finite $\Sigma$ - can be derived from [Má99, Theorem 2]. 
The next result deals with LCP sets of Banach algebra elements; it extends [Má98, Theorem 1] obtained for a finite family $\Sigma$ of linear bounded operators on a Hilbert space. As in Má98, we consider here some selected infinite products. Theorem 5.1 could also be formulated in this fashion.

Theorem 5.2. Let $\Sigma$ be a compact subset of a Banach algebra, and let $\mathcal{K}$ be a nonempty closed and shift-invariant subset of $\Sigma^{\infty}$. Set

$$
\hat{\rho}_{\mathcal{K}}(\Sigma):=\lim _{n \rightarrow \infty} \sup \left\{\left\|\sigma_{n} \cdots \sigma_{1}\right\|^{1 / n}:\left(\sigma_{1}, \ldots, \sigma_{n}\right) \in B_{n}(\mathcal{K})\right\} .
$$

The following statements are equivalent:

(i) $\hat{\rho}_{\mathcal{K}}(\Sigma)<1$;

(ii) for any $\sigma \in \mathcal{K}$, the series $\sum_{n=1}^{\infty} \sigma_{n} \cdots \sigma_{1}$ is absolutely convergent, and the set $\left\{\sum_{n=1}^{\infty} \sigma_{n} \cdots \sigma_{1}: \sigma \in \mathcal{K}\right\}$ is compact;

(iii) for any $\sigma \in \mathcal{K}, \lim _{n \rightarrow \infty} \sigma_{n} \cdots \sigma_{1}=0$;

(iv) for any $\sigma \in \mathcal{K}$, $\liminf _{n \rightarrow \infty}\left\|\sigma_{n} \cdots \sigma_{1}\right\|<1$.

Proof. Define $\Phi: B(\mathcal{K}) \rightarrow \mathbb{R}_{+}$by

$$
\Phi\left(\sigma_{1}, \ldots, \sigma_{n}\right):=\left\|\sigma_{n} \cdots \sigma_{1}\right\| \text { for } n \in \mathbb{N} \text { and }\left(\sigma_{1}, \ldots, \sigma_{n}\right) \in B_{n}(\mathcal{K}) .
$$

Then $\hat{\rho}_{\mathcal{K}}(\Sigma)=\rho(\Phi)$, so by Theorem 4.2 (i), (iii) and (iv) are equivalent. (ii) $\Rightarrow$ (iii) is clear. Since $\mathcal{K}$ is compact, a similar argument as in the proof of Theorem 5.1 ((i) $\Rightarrow($ ii)) shows (i) implies (ii).

\section{INFINITE PRODUCTS OF OPERATORS AND AN EXTENSION OF THE KELISKY-RIVLIN THEOREM}

We start with recalling Hutchinson's Hu81 theorem which is a fundamental result in the theory of iterated function systems.

Theorem 6.1 (Hutchinson). Let $T_{1}, \ldots, T_{N}$ be Banach contractions on a complete metric space $(X, d)$. For $\sigma \in\{1, \ldots, N\}^{\mathbb{N}}, n \in N$ and $x \in X$, set

$$
\Gamma(\sigma, n, x):=T_{\sigma_{1}} \circ \cdots \circ T_{\sigma_{n}} x .
$$

Then the limit $\Gamma(\sigma):=\lim _{n \rightarrow \infty} \Gamma(\sigma, n, x)$ exists and does not depend on $x$. Moreover, if $A$ is any bounded subset of $X$, then $(\Gamma(\sigma, n, x))_{n \in \mathbb{N}}$ converges to $\Gamma(\sigma)$ uniformly with respect to $x \in A$.

Theorem 6.1 prompts the following.

Definition 6.1. A family $\left\{T_{1}, \ldots, T_{N}\right\}$ of self-maps of a metric space $(X, d)$ is called a Hutchinson system if given any bounded subset $A$ of $X$, the sequence $(\Gamma(\sigma, n, x))_{n \in \mathbb{N}}$ defined by (6.1) converges to some $\Gamma(\sigma)$ for any $\sigma \in\{1, \ldots, N\}^{\mathbb{N}}$, uniformly with respect to $x \in A$.

Remark 6.1. For $N=1$, Definition 6.1 coincides with the notion of a super-regular mapping introduced by Reich and Zaslavski (see, e.g., [RZ01, p. 559]).

It is well known that if $T: X \rightarrow X$ is a mapping such that for some $p \in \mathbb{N}$ and $x_{*} \in X, \lim _{n \rightarrow \infty} T^{p n} x=x_{*}$ whenever $x \in X$, then also $\lim _{n \rightarrow \infty} T^{n} x=x_{*}$ for any $x \in X$. By [G-ŁJ05, Theorem 5], this result can be generalized to a finite family of mappings. Now we give its counterpart for Hutchinson systems. 
Proposition 6.1. Let $(X, d)$ be a metric space, and let $T_{1}, \ldots, T_{N}: X \rightarrow X$ be Lipschitzian mappings. If there exists $p \in \mathbb{N}$ such that the family

$$
\mathfrak{F}:=\left\{T_{i_{1}} \circ \cdots \circ T_{i_{p}}: i_{1}, \ldots, i_{p} \in\{1, \ldots, N\}\right\}
$$

is a Hutchinson system, so is $\left\{T_{1}, \ldots, T_{N}\right\}$.

Proof. If $p=1$, then we are done. So we assume $p \geq 2$. Let $A \subseteq X$ be bounded. Set

$$
B:=A \cup \bigcup\left\{\left(T_{i_{1}} \circ \cdots \circ T_{i_{k}}\right)(A): k=1, \ldots, p-1 ; i_{1}, \ldots, i_{k} \in\{1, \ldots, N\}\right\} .
$$

Then $B$ is bounded since all the sets $T_{i_{1}} \circ \cdots \circ T_{i_{k}}(A)$ are bounded because of the Lipschitz condition for $T_{1}, \ldots, T_{N}$. Since card $\mathfrak{F} \leq N^{p}$, we may draw up elements of $\mathfrak{F}$ in a sequence $\left(S_{i}\right)_{i=1}^{N^{p}}$.

Now fix $\sigma \in\{1, \ldots, N\}^{\mathbb{N}}$. For any $n \in \mathbb{N}$, there is $\sigma_{n}^{\prime} \in\left\{1, \ldots, N^{p}\right\}$ such that

$$
T_{\sigma_{(n-1) p+1}} \circ \cdots \circ T_{\sigma_{n p}}=S_{\sigma_{n}^{\prime}} .
$$

Define $\sigma^{\prime}:=\left(\sigma_{n}^{\prime}\right)_{n \in \mathbb{N}}$. By hypothesis, if

$$
\Gamma_{p}\left(\sigma^{\prime}, n, x\right):=S_{\sigma_{1}^{\prime}} \circ \cdots \circ S_{\sigma_{n}^{\prime}} x \text { for } n \in \mathbb{N} \text { and } x \in X,
$$

then $\lim _{n \rightarrow \infty} \Gamma_{p}\left(\sigma^{\prime}, n, x\right)=\Gamma_{p}\left(\sigma^{\prime}\right)$ uniformly with respect to $x \in B$. Thus, given $\varepsilon>0$, there is $k \in \mathbb{N}$ such that

$$
d\left(\Gamma_{p}\left(\sigma^{\prime}, n, x\right), \Gamma_{p}\left(\sigma^{\prime}\right)\right)<\varepsilon \text { for } n \geq k \text { and } x \in B .
$$

Let $n \geq k$ and $x \in A$. Since $A \subseteq B$ and $\Gamma_{p}\left(\sigma^{\prime}, n, x\right)=\Gamma(\sigma, n p, x)$, (6.2) yields

$$
d\left(\Gamma(\sigma, n p, x), \Gamma_{p}\left(\sigma^{\prime}\right)\right)<\varepsilon .
$$

Now, for any

$$
j=1, \ldots, p-1, T_{\sigma_{n p+1}} \circ T_{\sigma_{n p+2}} \circ \cdots \circ T_{\sigma_{n p+j}} x \in B
$$

and

so (6.2) gives

$$
\Gamma_{p}\left(\sigma^{\prime}, n, T_{\sigma_{n p+1}} \circ \cdots \circ T_{\sigma_{n p+j}} x\right)=\Gamma(\sigma, n p+j, x),
$$

$$
d\left(\Gamma(\sigma, n p+j, x), \Gamma_{p}\left(\sigma^{\prime}\right)\right)<\varepsilon .
$$

Thus the above argument shows that for any $n \geq k p$ and $x \in A$,

$$
d\left(\Gamma(\sigma, n, x), \Gamma_{p}\left(\sigma^{\prime}\right)\right)<\varepsilon
$$

This means $\lim _{n \rightarrow \infty} \Gamma(\sigma, n, x)=\Gamma(\sigma)$ uniformly with respect to $x \in A$, where $\Gamma(\sigma):=\Gamma_{p}\left(\sigma^{\prime}\right)$, so $\left\{T_{1}, \ldots, T_{N}\right\}$ is a Hutchinson system.

Lemma 6.1. Let $X$ be a linear space and $X_{0}$ be a subspace of $X$. Let $T: X \rightarrow X$ be a linear operator and $I$ be the identity mapping on $X$. The following statements are equivalent:

(i) every coset of the quotient space $X / X_{0}$ is T-invariant, i.e., $T\left(x+X_{0}\right) \subseteq$ $x+X_{0}$ for any $x \in X$;

(ii) $(I-T)(X) \subseteq X_{0}$.

Proof. (i) $\Rightarrow$ (ii): Fix $x \in X$. By (i), $T x=T(x+\Theta) \in x+X_{0}$, so $x-T x \in X_{0}$. Since $x$ was arbitrary, (ii) holds.

(ii) $\Rightarrow$ (i): Fix $x \in X$ and $x_{0} \in X_{0}$. Then we have

$$
T\left(x+x_{0}\right)=x-(x-T x)-\left(x_{0}-T x_{0}\right)+x_{0} \in x+X_{0}
$$

since, by (ii), $x-T x, x_{0}-T x_{0} \in X_{0}$. This yields $T\left(x+X_{0}\right) \subseteq x+X_{0}$. 
We are ready to give a necessary and sufficient condition for a finite family of linear bounded operators to be a Hutchinson system on every coset of a quotient space.

Theorem 6.2. Let $X$ be a Banach space and $X_{0}$ be a closed subspace of $X$. Let $T_{1}, \ldots, T_{N}: X \rightarrow X$ be linear bounded operators. The following statements are equivalent:

(i) for any $x \in X$, the family $\left\{\left.T_{1}\right|_{x+X_{0}}, \ldots,\left.T_{N}\right|_{x+X_{0}}\right\}$ is a Hutchinson system;

(ii) $\left(I-T_{i}\right)(X) \subseteq X_{0}$ for $i=1, \ldots, N$, and $\hat{\rho}\left(\left\{\left.T_{1}\right|_{X_{0}}, \ldots,\left.T_{N}\right|_{X_{0}}\right\}\right)<1$.

Proof. (i) $\Rightarrow$ (ii): By hypothesis, given $x \in X$ and $i \in\{1, \ldots, N\}, x+X_{0}$ is $T_{i^{-}}$ invariant which, in view of Lemma 6.1, yields $\left(I-T_{i}\right)(X) \subseteq X_{0}$. Since, in particular, $\left\{\left.T_{1}\right|_{X_{0}}, \ldots,\left.T_{N}\right|_{X_{0}}\right\}$ is a Hutchinson system, we infer $T_{i}\left(X_{0}\right) \subseteq X_{0}$ for $i=1, \ldots, N$, and given $\sigma \in\{1, \ldots, N\}^{\mathbb{N}},\left(T_{\sigma_{1}} \circ \cdots \circ T_{\sigma_{n}} x\right)_{n \in \mathbb{N}}$ converges to some $\Gamma(\sigma)$ uniformly with respect to $x$ from the unit ball in $X_{0}$; moreover, $\Gamma(\sigma)=\lim _{n \rightarrow \infty} T_{\sigma_{1}} \circ \cdots \circ$ $T_{\sigma_{n}} \Theta=\Theta$. This means

$$
\left\|\left.\left.T_{\sigma_{1}}\right|_{X_{0}} \circ \cdots \circ T_{\sigma_{n}}\right|_{X_{0}}\right\| \rightarrow 0,
$$

so Theorem [5.1 (with $\Sigma:=\left\{\left.T_{1}\right|_{X_{0}}, \ldots,\left.T_{N}\right|_{X_{0}}\right\}$ ) yields the second part of (ii).

(ii) $\Rightarrow($ i): By Lemma 6.1, the first part of (ii) implies every coset of the quotient space $X / X_{0}$ is $T_{i}$-invariant for $i=1, \ldots, N$. The second part of (ii) yields the existence of $p \in \mathbb{N}$ and $\alpha \in(0,1)$ such that

$$
\left\|\left.\left(T_{i_{1}} \circ \cdots \circ T_{i_{p}}\right)\right|_{X_{0}}\right\| \leq \alpha \text { for } i_{1}, \ldots, i_{p} \in\{1, \ldots, N\} .
$$

Fix $x \in X$. We show

$$
\mathfrak{F}:=\left\{\left.\left(T_{i_{1}} \circ \cdots \circ T_{i_{p}}\right)\right|_{x+X_{0}}: i_{1}, \ldots, i_{p} \in\{1, \ldots, N\}\right\}
$$

is the family of Banach contractions. Indeed, if $y_{1}, y_{2} \in x+X_{0}$, then, for some $x_{1}, x_{2} \in X_{0}, y_{j}=x+x_{j}$ for $j=1,2$, and hence we have

$$
\begin{aligned}
& \left\|T_{i_{1}} \circ \cdots \circ T_{i_{p}} y_{1}-T_{i_{1}} \circ \cdots \circ T_{i_{p}} y_{2}\right\|=\left\|T_{i_{1}} \circ \cdots \circ T_{i_{p}}\left(x_{1}-x_{2}\right)\right\| \\
& \quad \leq\left\|\left.\left(T_{i_{1}} \circ \cdots \circ T_{i_{p}}\right)\right|_{X_{0}}\right\| \cdot\left\|\left(x_{1}+x\right)-\left(x_{2}+x\right)\right\| \leq \alpha\left\|y_{1}-y_{2}\right\| .
\end{aligned}
$$

By Theorem 6.1 $\mathfrak{F}$ is a Hutchinson system, so it suffices to apply Proposition 6.1 to complete the proof.

As a particular case of Theorem 6.2, we obtain the following.

Corollary 6.1. Under the assumptions of Theorem 6.2, assume that $\|\left. T_{i}\right|_{X_{0}}||<1$ and $\left(I-T_{i}\right)(X) \subseteq X_{0}$ for $i=1, \ldots, N$. If $Y_{0}$ is a subspace of $X$ such that

$$
Y_{0} \subseteq \bigcap_{i=1}^{N} \operatorname{Fix} T_{i} \text { and } Y_{0} \cap\left(x+X_{0}\right) \neq \emptyset \text { for all } x \in X,
$$

then given $x \in X$ and $\sigma \in\{1, \ldots, N\}^{\mathbb{N}}$, the sequence $\left(T_{\sigma_{1}} \circ \cdots \circ T_{\sigma_{n}} x\right)_{n \in \mathbb{N}}$ is convergent and

$$
Y_{0} \cap\left(x+X_{0}\right)=\left\{\lim _{n \rightarrow \infty} T_{\sigma_{1}} \circ \cdots \circ T_{\sigma_{n}} x\right\} .
$$

Hence the above limit is independent of $\sigma$, but it may depend on $x$. 
Proof. By hypothesis and Lemma 2.2, we infer

$$
\hat{\rho}\left(\left\{\left.T_{1}\right|_{X_{0}}, \ldots,\left.T_{N}\right|_{X_{0}}\right\}\right) \leq \max \left\{\| T_{i}||: i=1, \ldots, N\right\}<1 .
$$

Fix $x \in X$. By Theorem 6.2 $\left\{\left.T_{1}\right|_{x+X_{0}}, \ldots,\left.T_{N}\right|_{x+X_{0}}\right\}$ is a Hutchinson system. Hence $\left.T_{1}\right|_{x+X_{0}}$ is super-regular (cf. Remark 6.1); in particular, Fix $\left.T_{1}\right|_{x+X_{0}}$ is a singleton. Hence and by hypothesis, we get

$$
\emptyset \neq Y_{0} \cap\left(x+X_{0}\right) \subseteq \operatorname{Fix} T_{1} \cap\left(x+X_{0}\right)=\left.\operatorname{Fix} T_{1}\right|_{x+X_{0}},
$$

which yields $Y_{0} \cap\left(x+X_{0}\right)=\{y\}$ for some $y \in X$. Since $x, y \in x+X_{0}$ and $y \in \bigcap_{i=1}^{N} \operatorname{Fix} T_{i}$, given $\sigma \in\{1, \ldots, N\}^{\mathbb{N}}$ we have

$$
\lim _{n \rightarrow \infty} T_{\sigma_{1}} \circ \cdots \circ T_{\sigma_{n}} x=\lim _{n \rightarrow \infty} T_{\sigma_{1}} \circ \cdots \circ T_{\sigma_{n}} y=y,
$$

which yields (6.3).

Remark 6.2. For $N=1$, Corollary [6.1 was proved in [Ja07 with the help of a version of the contraction principle for mappings on a metric space with a graph.

Corollary 6.1 unifies and generalizes many results in the literature concerning iterates of some linear bounded operators. In particular, it yields the following extension of the Kelisky-Rivlin KR67 theorem.

Theorem 6.3. For $n \in \mathbb{N}$, let $B_{n}$ be the Bernstein operator on the Banach space $C[0,1]$, i.e., for $f \in C[0,1]$ and $t \in[0,1]$,

$$
\left(B_{n} f\right)(t):=\sum_{k=0}^{n}\left(\begin{array}{l}
n \\
k
\end{array}\right) f\left(\frac{k}{n}\right) t^{k}(1-t)^{n-k} .
$$

Let $N$ be a positive integer. Then, for any $\sigma \in\{1, \ldots, N\}^{\mathbb{N}}$ and $f \in C[0,1]$,

$$
\lim _{n \rightarrow \infty}\left(B_{\sigma_{1}} \circ \cdots \circ B_{\sigma_{n}} f\right)(t)=f(0)+(f(1)-f(0)) t,
$$

uniformly with respect to $t \in[0,1]$. In particular,

$$
\lim _{n \rightarrow \infty}\left(B_{N}^{n} f\right)(t)=f(0)+(f(1)-f(0)) t \text { for } t \in[0,1] .
$$

Proof. Set $X:=C[0,1]$ and $X_{0}:=\{f \in X: f(0)=f(1)=0\}$. We show $\left\{B_{1}, \ldots, B_{N}\right\}$ satisfies the assumptions of Corollary 6.1. Given $i \in\{1, \ldots, N\}$, $f \in X$ and $t \in[0,1]$, we have

$$
\left(\left(I-B_{i}\right) f\right)(t)=\sum_{k=0}^{i}\left(\begin{array}{l}
i \\
k
\end{array}\right)\left(f(t)-f\left(\frac{k}{i}\right)\right) t^{k}(1-t)^{i-k} .
$$

Hence it is clear that $\left(I-B_{i}\right) f \in X_{0}$. We show $\left\|\left.B_{i}\right|_{X_{0}}\right\|<1$. If $f \in X_{0}$ and $t \in[0,1]$, then for $i \geq 2$,

$$
\begin{aligned}
\left|\left(B_{i} f\right)(t)\right| & =\left|\sum_{k=1}^{i-1}\left(\begin{array}{l}
i \\
k
\end{array}\right) f(k / i) t^{k}(1-t)^{i-k}\right| \\
& \leq\|f\|\left(1-(1-t)^{i}-t^{i}\right) \leq\|f\|\left(1-1 / 2^{i-1}\right),
\end{aligned}
$$

whereas for $i=1,\left(B_{i} f\right)(t)=0$. Thus we may infer

$$
\left.|| B_{i}\right|_{X_{0}} \| \leq 1-1 / 2^{i-1}<1 \text { for } i=1, \ldots, N .
$$


We define a space $Y_{0}$. It is well known that if $e_{i}(t):=t^{i}$ for $i=0,1$ and $t \in[0,1]$, then $B_{n} e_{i}=e_{i}$ for $n \in \mathbb{N}$. Hence, if $Y_{0}:=\operatorname{span}\left(e_{0}, e_{1}\right)$, then $Y_{0} \subseteq \bigcap_{i=1}^{N}$ Fix $B_{i}$. (In fact, the equality holds.) Moreover, given $f \in X$,

$$
f(0) e_{0}+(f(1)-f(0)) e_{1} \in Y_{0} \cap\left(f+X_{0}\right) .
$$

Hence and by Corollary 6.1, we infer that $\lim _{n \rightarrow \infty} B_{\sigma_{1}} \circ \cdots \circ B_{\sigma_{n}} f=f(0) e_{0}$ $+(f(1)-f(0)) e_{1}$ for any $\sigma \in\{1, \ldots, N\}^{\mathbb{N}}$. In particular, if $\sigma_{n}:=N$ for $n \in \mathbb{N}$, then we get (6.4).

Remark 6.3. The Kelisky-Rivlin theorem states only that (6.4) holds.

Finally, let us notice that in a similar way - with the help of Corollary [6.1 one can obtain a generalization of a recent result due to Oruç and Tuncer OT02, Theorem 3.1] concerning iterates of $q$-Bernstein polynomials introduced by Phillips Ph97.

\section{ACKNOWLEDGEMENT}

The author is grateful to the referee for some valuable remarks.

\section{REFERENCES}

[DL92] I. Daubechies and J. C. Lagarias, Sets of matrices all infinite products of which converge, Linear Algebra Appl. 161 (1992), 227-263. MR.1142737 (93f:15006)

[DL01] I. Daubechies and J. C. Lagarias, Corrigendum/addendum to: "Sets of matrices all infinite products of which converge", Linear Algebra Appl. 327 (2001), 69-83. MR.1823340 (2002b:15010)

[En77] R. Engelking, General Topology, Mathematical Monographs, Vol. 60, PWN-Polish Scientific Publishers, Warsaw, 1977. MR0500780 (58:18316b)

[G-ŁJ05] G. Gwóźdź-Łukawska and J. Jachymski, The Hutchinson-Barnsley theory for infinite iterated function systems, Bull. Austral. Math. Soc. 72 (2005), 441-454. MR2199645 (2006k:37042)

[HP57] E. Hille and R. S. Phillips, Functional Analysis and Semi-Groups, revised edition, American Mathematical Society Colloquium Publications, vol. 31, American Mathematical Society, Providence, R. I., 1957. MR.0089373 (19:664d)

[Hu81] J. E. Hutchinson, Fractals and self-similarity, Indiana Univ. Math. J. 30 (1981), 713747. MR625600 (82h:49026)

[Ja07] J. Jachymski, The contraction principle for mappings on a metric space with a graph, Proc. Amer. Math. Soc. 136 (2008), 1359-1373. MR.2367109

[KR67] R. P. Kelisky and T. J. Rivlin, Iterates of Bernstein polynomials, Pacific J. Math. 21 (1967), 511-520. MR0212457 (35:3328)

[Má98] L. Máté, On the infinite product of operators in Hilbert space, Proc. Amer. Math. Soc. 126 (1998), 535-543. MR.1415333 (98e:47002)

[Má99] L. Máté, On infinite composition of affine mappings, Fund. Math. 159 (1999), 85-90. MR.1669710 (99m:28043)

[OT02] H. Oruç and N. Tuncer, On the convergence and iterates of $q$-Bernstein polynomials, J. Approx. Theory 117 (2002), 301-313. MR.1924655 (2003h:41018)

[Ph97] G. M. Phillips, Bernstein polynomials based on the q-integers, Ann. Numer. Math. 4 (1997), 511-518. MR 1422700(97k:41013)

[RZ01] S. Reich and A. J. Zaslavski, Generic aspects of metric fixed point theory, in: Handbook of Metric Fixed Point Theory, W. A. Kirk and B. Sims (eds.), Kluwer Acad. Publ., Dordrecht, 2001, 557-575. MR1904287 (2003e:54048)

[RS60] G.-C. Rota and G. Strang, A note on the joint spectral radius, Indag. Math. 22 (1960), 379-381. MR0147922(26:5434)

[Sh00] J. Shen, Compactification of a set of matrices with convergent infinite products, Linear Algebra Appl. 311 (2000), 177-186. MR.1758212 (2001c:15039) 
[Va84] J. E. Vaughan, Countably compact and sequentially compact spaces, in: Handbook of Set-Theoretic Topology, K. Kunen and J. E. Vaughan (eds.), North-Holland, Amsterdam, 1984, 569-602. MR776631 (86c:54022)

[Yo80] K. Yosida, Functional Analysis, Grundlehren Math. Wiss. 123, Springer, Berlin, 1980. MR617913(82i:46002)

Institute of Mathematics, Technical University of Łódź, Wólczańska 215, 93-005 Łódź, POLAND

E-mail address: jachym@p.lodz.pl 